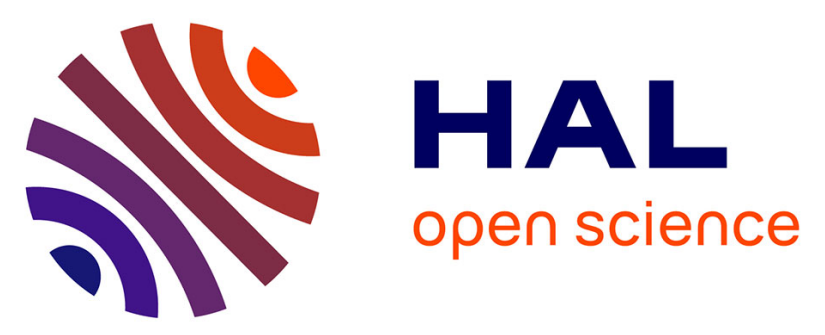

\title{
Non-steady-state photoelectromotive force and two-wave mixing in photorefractive crystals under frequency modulated illumination
}

\author{
M. Bryushinin, V. Kulikov, I. Sokolov, Philippe Delaye, Gilles Pauliat
}

\section{- To cite this version:}

M. Bryushinin, V. Kulikov, I. Sokolov, Philippe Delaye, Gilles Pauliat. Non-steady-state photoelectromotive force and two-wave mixing in photorefractive crystals under frequency modulated illumination. Journal of the Optical Society of America B, 2014, 31 (4), pp.723. 10.1364/JOSAB.31.000723 . hal00997138

\section{HAL Id: hal-00997138 \\ https://hal-iogs.archives-ouvertes.fr/hal-00997138}

Submitted on 27 May 2014

HAL is a multi-disciplinary open access archive for the deposit and dissemination of scientific research documents, whether they are published or not. The documents may come from teaching and research institutions in France or abroad, or from public or private research centers.
L'archive ouverte pluridisciplinaire HAL, est destinée au dépôt et à la diffusion de documents scientifiques de niveau recherche, publiés ou non, émanant des établissements d'enseignement et de recherche français ou étrangers, des laboratoires publics ou privés. 


\title{
Non-steady-state photoelectromotive force and two-wave mixing in photorefractive crystals under frequency modulated illumination
}

\author{
M. Bryushinin, ${ }^{1, *}$ V. Kulikov, ${ }^{1}$ I. Sokolov, ${ }^{1}$ P. Delaye,${ }^{2}$ and G. Pauliat ${ }^{2}$ \\ ${ }^{1}$ Ioffe Physical Technical Institute, Politekhnicheskaya 26, 194021 St. Petersburg, Russia \\ ${ }^{2}$ Laboratoire Charles Fabry, Institut d'Optique, CNRS, Univ Paris-Sud, 2 av. A. Fresnel, 91127 Palaiseau cedex, France \\ ${ }^{*}$ Corresponding author: mb@mail.ioffe.ru
}

Received January 2, 2014; accepted January 23, 2014;

posted February 7, 2014 (Doc. ID 203875); published March 7, 2014

\begin{abstract}
We report the excitation of non-steady-state photoelectromotive force and two-wave mixing signals using uniformly accelerated motion of the recording light pattern. Such illumination is created by linear frequency modulation of the interfering light beams. The pulse response is predicted theoretically and observed experimentally in GaAs and $\mathrm{Bi}_{12} \mathrm{TiO}_{20}$ crystals at $\lambda=633 \mathrm{~nm}$. The evolution of the pulse shape versus sweep rate is demonstrated and explained in the frames of the developed theory. The application of the effects in laser Doppler velocimeters and accelerometers is discussed as well. (c) 2014 Optical Society of America

OCIS codes: (190.5330) Photorefractive optics; (190.2055) Dynamic gratings; (160.5320) Photorefractive

materials; (280.3340) Laser Doppler velocimetry.

http://dx.doi.org/10.1364/JOSAB.31.000723
\end{abstract}

\section{INTRODUCTION}

The evolution of photoinduced space charge under nonuniform and nonstationary illumination gives rise to a number of nonlinear effects in photorefractive media: two- and four-wave mixing, space-charge waves, non-steady-state photoelectromotive force (photo-EMF), etc. [1,2]. Some of them have been observed with a running or oscillating interference pattern. Such patterns are created by two coherent light beams shifted by frequency or phase modulated by a sinusoidal signal, respectively. Utilization of a light pattern running with constant velocity usually results in a dc output signal, while the techniques using an oscillating pattern provide an ac one. Constant frequency shift can arise because of the Doppler effect, and sinusoidal phase modulation appears in the beam reflected from a vibrating object. Corresponding schemes of velocimeters and vibrometers using photorefractive materials have been proposed [3-11].

In this study we try to extend the variety of the effects observed in photorefractive and photoconductive media introducing linear frequency modulation (LFM) in the light beams forming an interference pattern. We investigate theoretically and experimentally the manifestation of such illumination in the non-steady-state photo-EMF and two-wave mixing effects. The conventional effect of non-steady-state photo-EMF reveals itself as an alternating current arising in a semiconductor illuminated by an oscillating interference pattern [12-14]. The electric current appears due to spatial shifts of the spacecharge and photoconductivity distributions in the crystal volume. The related techniques realized with an interference pattern running with constant velocity are called the holographic current [15] or moving photocarrier grating technique [16]. The two-wave mixing effect has been studied for years in photorefractive crystals and consists in intensity or phase redistribution for light waves propagating in the direction of the signal and reference beams [17-21]. In the case of an oscillating interference pattern, the amplitudes and phases of these waves become time dependent because of the periodic spatial mismatch of the light intensity and refractive index distributions. In the case of a running light pattern they observe a dc two-wave mixing signal (stationary changes of light intensity behind the crystal). The excitation of both the photo-EMF and wave mixing signals involves processes of carrier generation, diffusion and drift in an electric field, and recombination to local centers. This complex nature of the effects makes them powerful tools for determination of photoelectric parameters, such as type and value of photoconductivity, carrier lifetime, mobility and diffusion length, and density of recombination centers [14,16,22-25].

One can expect that LFM will result in pulsed response. Indeed, the response of the highly resistive material to initial fast variations of light intensity is negligibly small. At the moment when the interference pattern slows down and stops, a noticeable space-charge grating is recorded, and signals can appear. Then the pattern begins to run fast again and signals vanish. In the following sections we consider this effect in detail and discuss its possible applications.

\section{THEORETICAL ANALYSIS}

The main idea of the experiment is sketched in Fig. 1 . The crystal is illuminated by two light waves:

$$
\begin{gathered}
E_{R}(\mathbf{r}, t)=\left(E_{R} / 2\right) \exp \left[i \mathbf{k}_{\mathbf{R}} \mathbf{r}-i \psi_{R}(t)\right]+\text { c.c. }, \\
E_{S}(\mathbf{r}, t)=\left(E_{S} / 2\right) \exp \left[i \mathbf{k}_{\mathbf{S}} \mathbf{r}-i \psi_{S}(t)\right]+\text { c.c. }
\end{gathered}
$$




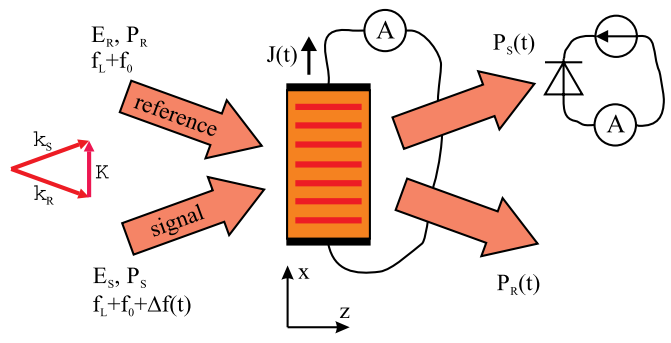

Fig. 1. Scheme of the space-charge formation and excitation of the non-steady-state photo-EMF and two-wave mixing signals by frequency modulated light.

Both waves are polarized perpendicular to the incidence plane; the incidence angles are equal by modulus. These waves are frequency detuned by frequency shifters:

$$
\begin{gathered}
\psi_{R}=2 \pi\left(f_{L}+f_{0}\right) t \\
\psi_{S}=2 \pi\left(f_{L}+f_{0}\right) t+2 \pi \int_{0}^{t} \Delta f\left(t^{\prime}\right) \mathrm{d} t^{\prime},
\end{gathered}
$$

where $f_{L}=c / \lambda$ is the frequency of light, $f_{0}$ is the initial frequency shift ( $\sim 80-120 \mathrm{MHz}$ for acousto-optic modulators), and $\Delta f(t)$ is the frequency shift in the signal beam, which results in signal appearance. We consider the case of LFM:

$$
\Delta f(t)=A t .
$$

We should note that frequency shift by an acousto-optic modulator can be accompanied by beam deflection: in our case this means that vector $\mathbf{k}_{\mathbf{s}}$ can change not only its modulus but also the direction. However, we assume the frequency shifters to be ideal, operating without beam deflection.

These beams produce the running interference pattern:

$$
I(x, t)=I_{0}\{1+m \cos [K x+\varphi(t)]\},
$$

where $I_{0}$ is the average intensity, $m$ and $K$ are the contrast and spatial frequency of the interference pattern, and

$$
\varphi(t)=-2 \pi \int_{0}^{t} \Delta f\left(t^{\prime}\right) \mathrm{d} t^{\prime}=-\pi A t^{2},
$$

is the phase of the interference pattern. In fact, grating vector $\mathbf{K}=\mathbf{k}_{\mathbf{S}}-\mathbf{k}_{\mathbf{R}}$ and corresponding spatial frequency $K=(\mathbf{K})_{x}$ are time dependent since $\mathbf{k}_{\mathbf{S}}=\mathbf{k}_{\mathbf{S}}(t)$. We neglect these variations as they are very small: $|\Delta K| / K \simeq|\Delta f(t)| / 2 f_{L} \leq 10^{-8}$ for sensible frequency shifts $|\Delta f(t)| \leq 10 \mathrm{MHz}$ and not very small angles between beams (0.1-1 rad). Expression (6) can be rewritten in a form more convenient for calculations of the space-charge field:

$$
I(x, t)=I_{0}\left[1+\frac{m(t)}{2} e^{i K x}+\frac{m^{*}(t)}{2} e^{-i K x}\right],
$$

where

$$
m(t)=m \exp [i \varphi(t)]
$$

Further analysis is performed for the simplest model of electron semiconductor with one type of partially compensated donor centers [2]. We consider the diffusion mechanism of space-charge formation, which takes place in the absence of an external electric field. The following assumptions are applied as usual: the excitation and recombination of electrons are linear, dark conductivity and the photovoltaic effect are absent, and contrast $m$ and light absorption are small. Then distributions of the density of electrons $n(x, t)$ and space-charge field $E_{s c}(x, t)$ are sinusoidal and can be presented as follows [2]:

$$
\begin{gathered}
n(x, t)=n_{0}\left[1+\frac{a(t)}{2} e^{i K x}+\frac{a^{*}(t)}{2} e^{-i K x}\right], \\
E_{s c}(x, t)=\frac{E_{s c}(t)}{2} e^{i K x}+\frac{E_{s c}^{*}(t)}{2} e^{-i K x} .
\end{gathered}
$$

Complex amplitudes $a(t)$ and $E_{s c}(t)$ are found from the following set of linear differential equations, which are just rewritten material, continuity, and Poisson equations:

$$
\begin{gathered}
\frac{d a}{d t}=-\frac{1+K^{2} L_{D}^{2}}{\tau} a+i K \mu E_{s c}+\frac{m(t)}{\tau} \\
\frac{d E_{s c}}{d t}=-\frac{i E_{D}}{\tau_{M}} a-\frac{1}{\tau_{M}} E_{s c}
\end{gathered}
$$

Here $\mu, \tau$, and $L_{D}$ are the mobility, lifetime, and diffusion length of electrons, $\tau_{M}=\epsilon \epsilon_{0} / \sigma_{0}$ is the Maxwell relaxation time of the material with the average conductivity $\sigma_{0}=$ $e \mu n_{0}$ and dielectric constant $\epsilon$, and $E_{D}=\left(k_{B} T / e\right) K$ is the diffusion field.

One can easily write the solution of Eqs. (12) and (13), but it is reasonable to make another simplifying assumption: formation of the space charge occurs with the quasi-stationary distribution of electrons in the conduction band [2]; i.e., the electron lifetime $\tau$ is small compared to the characteristic time of changes in the light pattern, in our case $\tau \ll|\Delta f(t)|^{-1}$. Then the desired solution is transformed into rather compact formulae:

$$
\begin{gathered}
a(t)=\frac{m(t)+i \mu \tau K E_{s c}(t)}{1+K^{2} L_{D}^{2}}, \\
E_{s c}(t)=\frac{-i E_{D}}{\tau_{s c}} \int_{0}^{\infty} m\left(t-t^{\prime}\right) e^{-t^{\prime} / \tau_{s c}} \mathrm{~d} t^{\prime} .
\end{gathered}
$$

Now let us proceed with the calculation of the values measured in the experiment: photo-EMF and two-wave mixing signals. The photo-EMF signal is defined by the drift component of the photocurrent averaged over interelectrode spacing and presented as a combination of complex amplitudes $a(t)$ and $E_{s c}(t)[\underline{12}-\underline{14}]$ :

$$
j(t)=\sigma_{0} \operatorname{Re}\left[a(t) E_{s c}^{*}(t)\right] / 2,
$$

where Re means the real part. After necessary substitutions we obtain the expression for photo-EMF signal: 


$$
j(t)=-\frac{m^{2} \sigma_{0} E_{D}}{2\left(1+K^{2} L_{D}^{2}\right)} \operatorname{Im} \Xi(t),
$$

where Im means the imaginary part and

$$
\Xi(t)=\tau_{s c}^{-1} \int_{0}^{\infty} \exp \left\{i\left[\varphi(t)-\varphi\left(t-t^{\prime}\right)\right]-t^{\prime} / \tau_{s c}\right\} \mathrm{d} t^{\prime}
$$

The signal in two-wave mixing experiments is usually detected by a photodiode, whose current is proportional to the power of light emerging the crystal in the direction of the signal (or reference) beam. If we neglect the absorption and reflection of the light and consider the case of thin holograms, then the power of "signal" beam behind the crystal is as follows $[\underline{20,21]}$ :

$$
P_{S}(t)=P_{S}+P_{R} \delta^{2} d^{2}\left|E_{s c}(t)\right|^{2} / 4+\sqrt{P_{R} P_{S}} \delta d \operatorname{Im}\left[e^{i \varphi(t)} E_{s c}^{*}(t)\right],
$$

where $P_{R}$ and $P_{S}$ are the powers of the incident light in the reference and signal beams, $\delta=\pi n_{0}^{3} r / \lambda^{\prime} \cos \theta$ is the coefficient [18,19], $n_{0}$ is the refractive index, $r$ is the electro-optic coefficient, $\lambda^{\prime}=c /\left(f_{L}+f_{0}\right)$ is the wavelength of the light after the frequency shifter, $2 \theta$ is the angle between beams inside the crystal, and $d$ is the crystal thickness. The first term is the transmitted light of the signal beam, and it is time independent. The second term describes the light diffracted from the reference beam. It is weak because of the small diffraction efficiency of thin holograms: $\left|\delta d E_{s c} / 2\right| \ll 1$. The last term determines the main part of the two-wave mixing signal, and after necessary substitutions it equals

$$
P_{S \sim}(t)=P_{0} \frac{m^{2} \delta d E_{D}}{2} \operatorname{Re} \Xi(t),
$$

where $P_{0}=P_{R}+P_{S}$ is the total power of the incident light. The two-wave mixing signal in the "reference" beam behind the crystal has the opposite sign: $P_{R \sim}(t)=-P_{S \sim}(t)$.

Fortunately, for the considered LFM (7) integral (18) can be represented via a complementary error function:

$$
\begin{aligned}
\Xi(t)= & \frac{1}{2 \sqrt{A \tau_{s c}^{2}}} \exp \left[-\frac{t}{\tau_{s c}}+i\left(\frac{\pi}{4}+\frac{1}{4 \pi A \tau_{s c}^{2}}-\pi A t^{2}\right)\right] \\
& \times \operatorname{Erf}\left(-\sqrt{\frac{\pi A}{i}} t+\sqrt{\frac{i}{4 \pi A \tau_{s c}^{2}}}\right) .
\end{aligned}
$$

This allows us to calculate time dependencies of the photoEMF and two-wave mixing signals (Fig. 2). Having rewritten $\Xi$ for dimensionless time $t / \tau_{s c}$ one can easily note that this function has the only parameter $A \tau_{s c}^{2}$.

Solution (21) can be simplified for two extreme cases. The former corresponds to very slow frequency modulation $\left(\pi A \tau_{s c}^{2} \ll 1\right)$ :

$$
\Xi(t)=\left(1+i 2 \pi A t \tau_{s c}\right)^{-1}=\left(1+i 2 \pi \Delta f(t) \tau_{s c}\right)^{-1} .
$$

This result is exactly the same as that calculated for the case of constant frequency shift $\Delta f(t)=\Delta f_{c}=\operatorname{const}(t)$ [5] . This means, in particular, that frequency dependence measured
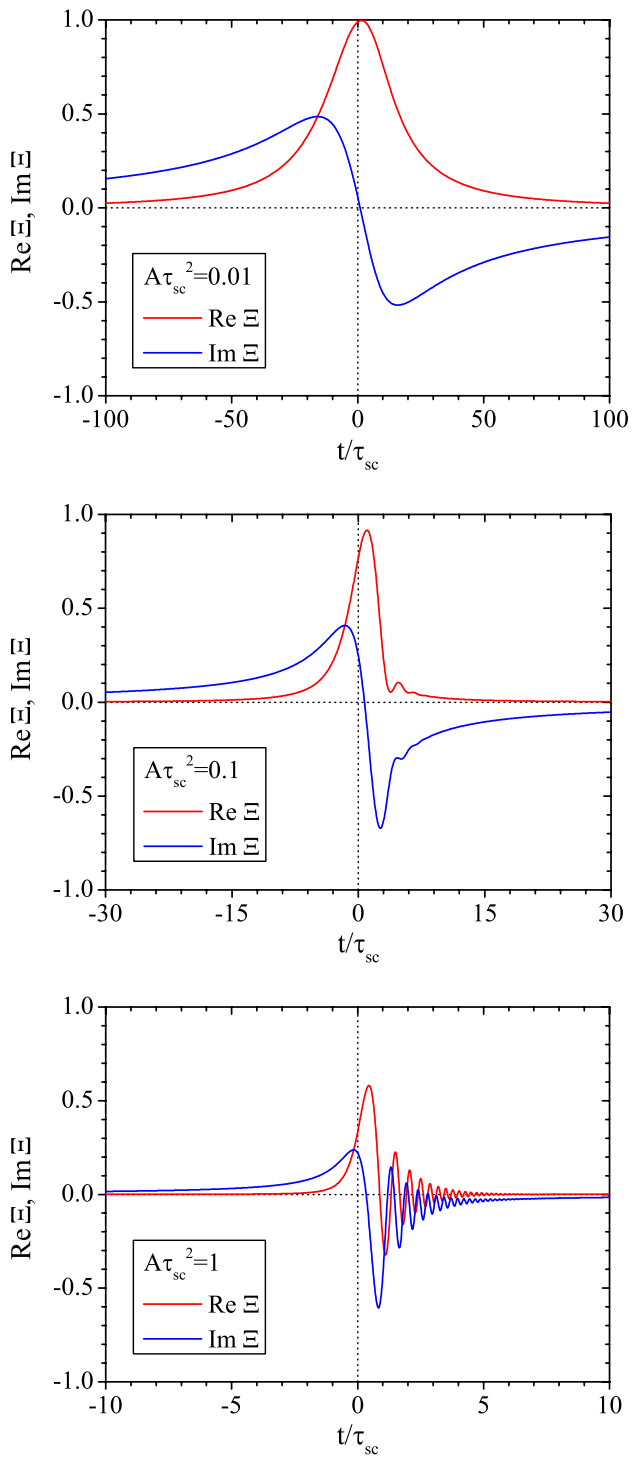

Fig. 2. Time dependencies of the real and imaginary parts of integral $\Xi$ (normalized two-wave mixing and photo-EMF signals) calculated for different sweep rates $A \tau_{s c}^{2}=0.01,0.1$, and 1 .

by a spectrum analyzer (with lowest sweep rate) coincides with the frequency dependence measured "point by point."

Another extreme case realizes at fast frequency modulation $\left(\pi A \tau_{s c}^{2} \gg 1\right)$. For $t<-\tau_{s c}$ function $\Xi(t)$ reduces to Eq. (22) as well. For $t>\tau_{s c}$ an additional oscillatory behavior appears:

$$
\begin{aligned}
\Xi(t)= & \left(1+i 2 \pi A t \tau_{s c}\right)^{-1}+\left(A \tau_{s c}^{2}\right)^{-1 / 2} \\
& \times \exp \left[-t / \tau_{s c}+i\left(\pi / 4-\pi A t^{2}\right)\right] .
\end{aligned}
$$

The appearance of oscillations can be simply explained. The interference pattern runs very fast at $t<0$ preventing formation of a space-charge field grating. Then it slows down and stops at $t=0$. The stationary field grating with a noticeable amplitude arises. At $0<t<\tau_{s c}$ the interference pattern begins to run in the opposite direction but the recorded stationary grating is still present. This combination of running interference pattern and stationary grating gives rise to oscillations of the current and light intensity analogous to the ones in experiments with conventional non-steady-state photo-EMF and 
two-wave mixing. These oscillations are decaying since the space-charge field grating erases with characteristic time $\tau_{s c}$.

Evaluating the real and imaginary parts of Eq. (22), one can note differences in their behavior at large $t$ (Fig. 2): the real part (two-wave mixing signal) decays as $t^{-2}$, while the imaginary one (photo-EMF signal) decays as $t^{-1}$.

\section{EXPERIMENTAL ARRANGEMENTS}

The experimental setup used for measurements of the nonsteady-state photo-EMF and two-wave mixing signals is shown in Fig. 3. The light from an He-Ne laser $(\lambda=633 \mathrm{~nm}$, $P_{\text {out }} \simeq 30 \mathrm{~mW}$ ) passes through two acousto-optic modulators ML-201-1, which introduce frequency shifts $f_{0}$ and $f_{0}+\Delta f(t)$ into diffracted light beams $\left(f_{0}=80 \mathrm{MHz}\right)$. We use the output of spectrum analyzer SK4-59 as a generator of the linear frequency modulated signal because of its wide range of frequency deviation. Then these beams are directed to the sample forming the interference pattern with average intensity $I_{0}=240 \mathrm{~mW} / \mathrm{cm}^{2}$ and contrast $m=0.97$ (the power of signal and reference beams is $P_{S}=3.4 \mathrm{~mW}$ and $P_{R}=2.0 \mathrm{~mW}$, respectively). The excitation of the non-steady-state photoEMF is carried out in semi-insulating GaAs crystal. The sample has dimensions of $3 \times \mathrm{mm} \times 3 \times \mathrm{mm} \times 0.5 \mathrm{~mm}$; the front and back surfaces $3 \times \mathrm{mm} \times 3 \mathrm{~mm}$ were polished. Two gold stripes were deposited on the front surface (with interelectrode spacing of $1 \mathrm{~mm}$ ). The sample is tilted around the direction of light propagation by $\sim 15^{\circ}$ in order to decrease contact photo-EMF [26]. The non-steady-state photo-EMF creates voltage on the load resistor $R_{L}=1 \mathrm{M} \Omega$, which is measured by the digital oscilloscope. The experiments on two-wave mixing are performed with $\mathrm{Bi}_{12} \mathrm{TiO}_{20}$ undoped crystal $(9.5 \mathrm{~mm} \times$ $12 \mathrm{~mm} \times 11 \mathrm{~mm})$. The sample has one of the standard holographic orientations: cut (110) with axis [001] lying in the incidence plane. The front and back surfaces $9.5 \mathrm{~mm} \times 12 \mathrm{~mm}$ were polished; no electrodes were deposited. Since the crystal is rather thick and rotates polarization by $\sim 70^{\circ}$, the half-wave plate is placed in front of it. As a result, the polarization plane in the middle of the crystal is settled perpendicular to the incident plane. The power of the light emerging from the crystal in the direction of the signal beam is measured by the photodiode and visualized by the oscilloscope. The sweep voltage from the spectrum analyzer feeds the oscilloscope as well

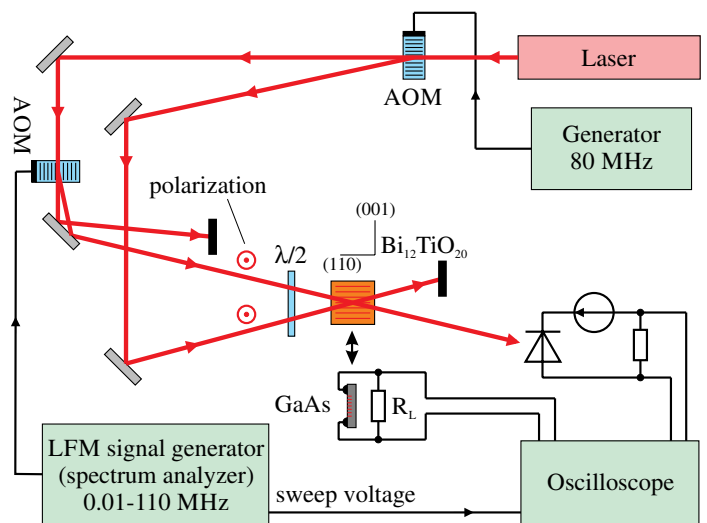

Fig. 3. Experimental setup used for measurements of the nonsteady-state photo-EMF and two-wave mixing signals excited by frequency modulated light. $A O M$ represents the acousto-optic modulators. The sample ( $\mathrm{GaAs}$ or $\mathrm{Bi}_{12} \mathrm{TiO}_{20}$ ) is placed in the intersection of light beams in the corresponding experiment. allowing simultaneous measurements of the frequency shift and investigated signal.

\section{EXPERIMENTAL RESULTS}

\section{A. Non-Steady-State Photo-EMF in GaAs}

First let us find whether the moment of signal appearance is unambiguously determined by the slowing down and stopping of the interference pattern, i.e., by the coincidence of frequencies of the signal and reference beams. We settle several different central frequencies on the spectrum analyzer (79.6-80.4 MHz) and measure corresponding traces of the non-steady-state photo-EMF (Fig. 4). As seen, the pulse position, i.e., the time when photocurrent changes its sign, well corresponds with the moments when frequencies of light waves are equal $(\Delta f=0)$.

Figure 5 presents traces of the non-steady-state photo-EMF signal measured at different sweep rates. The signal demonstrates qualitatively the same behavior as described in theoretical analysis. At low sweep rates the pulse consists of two parts with nearly equal amplitudes and opposite signs. The increase of sweep rate decreases the pulse duration, while the amplitude remains nearly constant. At large sweep rates the negative peak becomes sharper than the positive one. At very large $A$ chirped and damped oscillations appear. The most pronounced oscillations are achieved by simultaneous increase of sweep rate and decrease of illumination level (the pulse amplitude and corresponding signal-to-noise ratio become lower, however).

There are, however, some peculiarities that are not described by the developed theory. First, the pulse measured at lowest sweep rate $\left(A=10^{6} \mathrm{~Hz} / \mathrm{s}\right)$ should be the most symmetric. In practice the preceding positive half has 1.9 times smaller amplitude than the following negative one (trace is not shown in the figure). Second, the pulse tails decay slower than $t^{-1}$ as predicted by Eq. (22). The thin blue lines in Fig. $\underline{5}$ show the approximation by Eq. (21) for $\tau_{s c}=12 \mu \mathrm{s}$. The origin of these discrepancies is unknown; we can just suppose that it can be associated with bipolar conductivity and high light absorption in GaAs at $\lambda=633 \mathrm{~nm}$. Both factors sufficiently change the dynamics of space-charge formation and nonsteady-state photo-EMF characteristics [27-29].

We have also measured the dependence of the signal on the light intensity (Fig. 6). The peak-to-peak amplitude of the

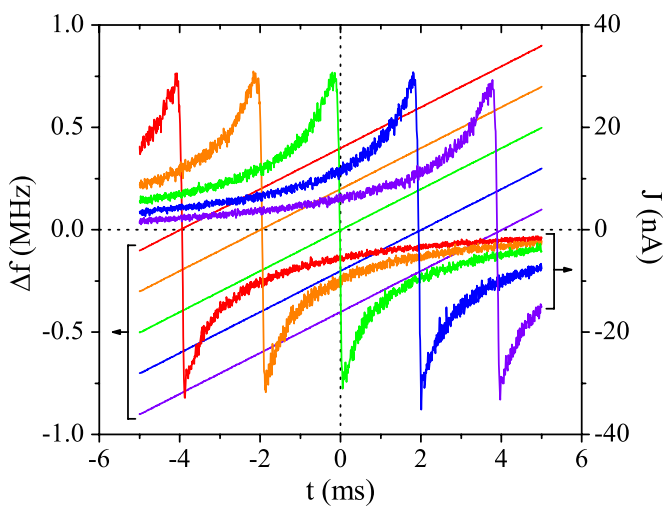

Fig. 4. Oscillograms of the frequency shift in signal beam and the corresponding current arising in GaAs crystal. $\lambda=633 \mathrm{~nm}$ $I_{0}=240 \mathrm{~mW} / \mathrm{cm}^{2}, K=190 \mathrm{~mm}^{-1}$, and $A=10^{8} \mathrm{~Hz} / \mathrm{s}$. 

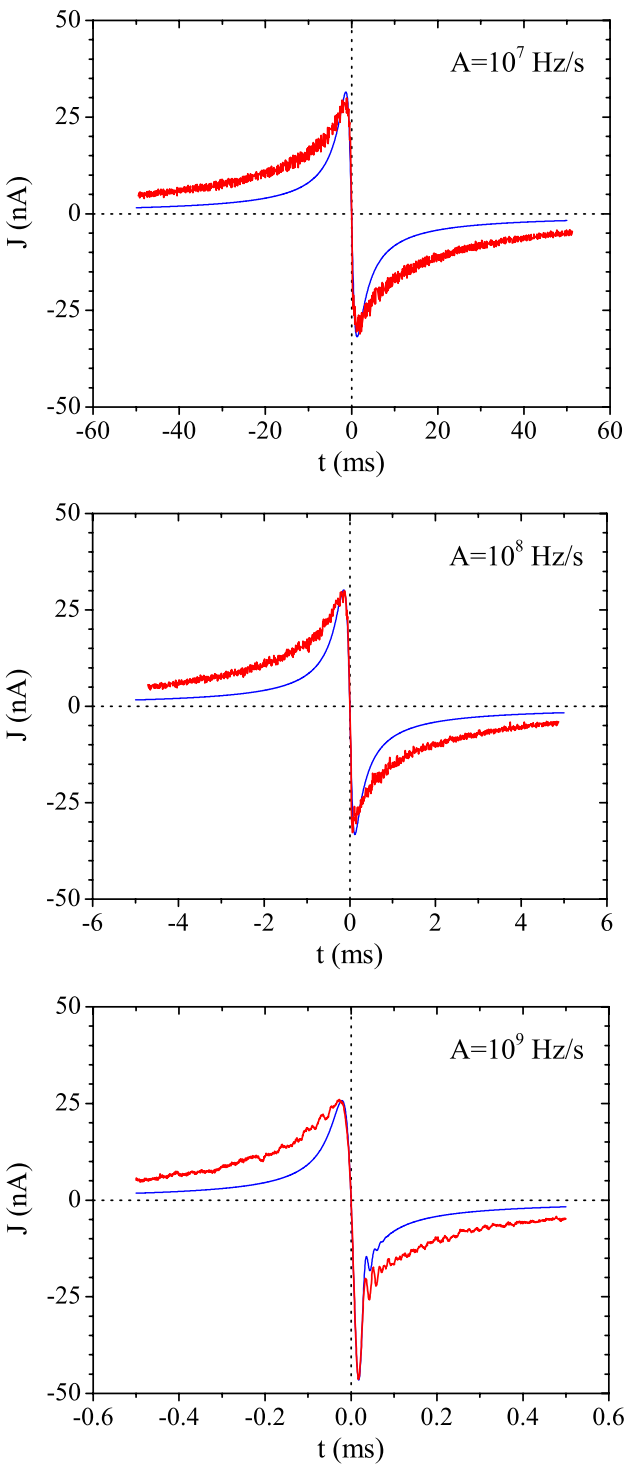

Fig. 5. Oscillograms of the photocurrent in GaAs crystal measured for different sweep rates $A=10^{6}, 10^{7}, 10^{8}$, and $10^{9} \mathrm{~Hz} / \mathrm{s} . \lambda=633 \mathrm{~nm}$, $I_{0}=240 \mathrm{~mW} / \mathrm{cm}^{2}$, and $K=190 \mathrm{~mm}^{-1}$. Thin blue lines show approximation by Eq. (21) for $\tau_{s c}=12 \mu \mathrm{s}$.

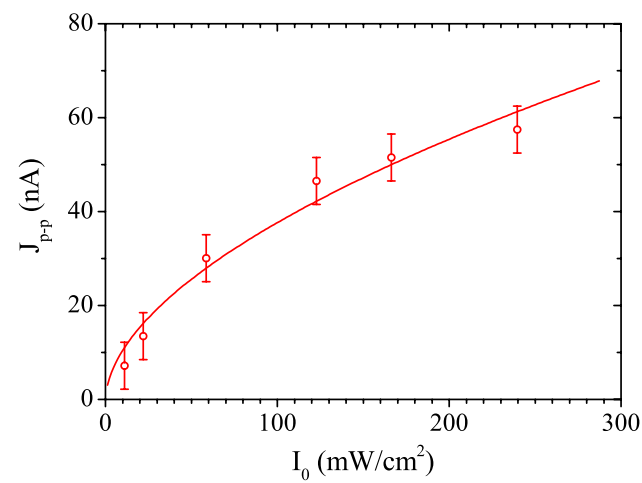

Fig. 6. Dependence of the photo-EMF peak-to-peak amplitude versus average light intensity. GaAs, $\lambda=633 \mathrm{~nm}, K=190 \mathrm{~mm}^{-1}$, and $A=5 \times 10^{7} \mathrm{~Hz} / \mathrm{s}$. Solid line shows approximation $J_{p-p} \propto I_{0}^{0.56}$. pulse demonstrates noticeable sublinearity $J_{p-p} \propto I_{0}^{0.56}$ in the range $I_{0}=11-240 \mathrm{~mW} / \mathrm{cm}^{2}$, which is rather typical for GaAs crystal at this wavelength. Decreasing the light intensity, we observed evolution of the pulse shape similar to that for increasing sweep rate. This fact confirms the statement that pulse shape is determined by parameter $A \tau_{s c}^{2}$.

\section{B. Two-Wave Mixing in $\mathbf{B i}_{12} \mathbf{T i O}_{20}$}

Traces of the two-wave mixing signals excited by LFM light waves are shown in Fig. 7. Chirped pulses are observed for sweep rates of $0.25-50 \mathrm{kHz} / \mathrm{s}$. Their behavior is well described by the developed theory: the pulses appear at the moments when the interference pattern stops, and the oscillations are frequency modulated and damped; their duration and amplitude decrease with increasing sweep rate. The signals are noisy; nevertheless, we can state that the pulse tails of two-wave mixing decay faster than the ones of photo-EMF (at least for $t<0$ ), as the theory predicts.

The measurements are carried out at sweep rates much lower than those for photo-EMF experiments. Small
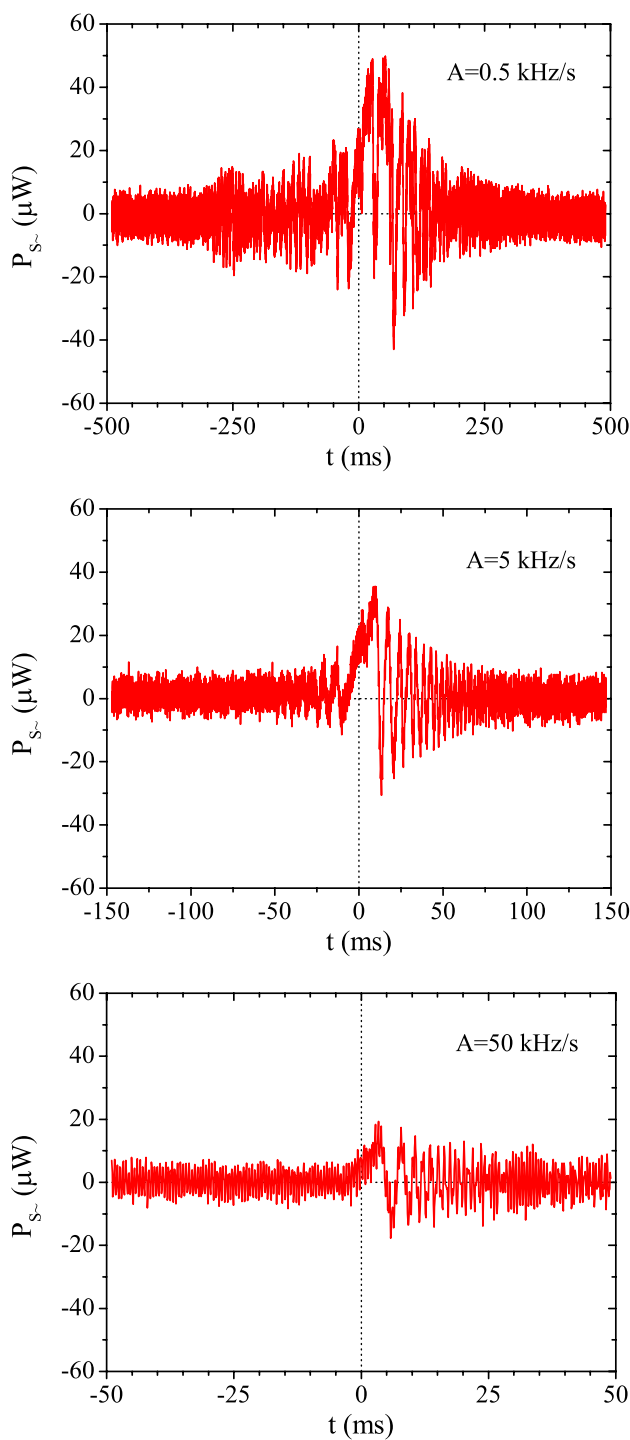

Fig. 7. Oscillograms of the two-wave mixing ac signal measured in $\mathrm{Bi}_{12} \mathrm{TiO}_{20}$ crystal at different sweep rates: $A=0.5,5$, and $50 \mathrm{kHz} / \mathrm{s}$. $\lambda=633 \mathrm{~nm}, I_{0}=240 \mathrm{~mW} / \mathrm{cm}^{2}$, and $K=2.3 \mu \mathrm{m}^{-1}$. 
photoconductivity of $\mathrm{Bi}_{12} \mathrm{TiO}_{20}$ crystals results in large time of space-charge formation $\tau_{s c} \sim 0.1 \mathrm{~s}$. This means that even the lowest used sweep rate of $250 \mathrm{~Hz} / \mathrm{s}$ satisfies the condition $\pi A \tau_{s c}^{2} \gg 1$, and that is why only oscillatory signals are detected. We can settle lower sweep rates, but the signal becomes very unstable: the used vibroisolation is not enough for pulse durations larger than $100 \mathrm{~ms}$.

We should note here that the theoretical analysis has been carried out for a thin hologram, while the two-wave mixing experiment is realized in geometry of a thick one $\left(\lambda d / n_{0} \Lambda^{2} \simeq 400 \gg 1\right.$, where $\left.\Lambda=2 \pi / K\right)$. We have to use thick crystal because the signals are rather small and detected in wide band $(5 \mathrm{kHz})$. We can estimate the maximal amplitude of the two-wave mixing signal from Eq. (20): $P_{0} m^{2} \delta d E_{D} / 2=$ $470 \mu \mathrm{W}$. As seen, this estimation exceeds the experimental value nearly by an order of magnitude. Several factors can be taken into account to explain this discrepancy: first, we measure only oscillating signals, while the maximum amplitude should be achieved at lower sweep rates; second, the approach of a thin hologram provides overrated diffraction efficiency for a thick one [2]; and third, there are noticeable light absorption and optical activity, which decrease the signal as well.

\section{DISCUSSION}

The results of theoretical analysis and experiments in GaAs and $\mathrm{Bi}_{12} \mathrm{TiO}_{20}$ generally match each other. The time of signal appearance, variation of the pulse duration and shape versus sweep rate and light intensity are well described by the theory. However, some features, such as pulse tails in GaAs and low pulse amplitude in $\mathrm{Bi}_{12} \mathrm{TiO}_{20}$, require more advanced theoretical approaches dealing with bipolar conductivity, high light absorption, and vectorial coupling [27-32].

For years we emphasized the fact that the frequency transfer function of the conventional non-steady-state photo-EMF measured at low frequencies is similar to that of the simplest differentiating RC circuit (high-pass filter) $[13,14]$. In the case of LFM signals this similarity vanishes: at large $|t|$ the frequency is large too, and ac voltage freely passes through the differentiating RC circuit; at low $|t|$ when the frequency is small the signal is damped. We can note that the behavior of the photo-EMF signal for LFM is more similar to the one of the integrating RC circuit (low-pass filter). Indeed, the voltage $\operatorname{Re}\left\{U_{0} \exp [i \varphi(t)]\right\}$ feeding the low-pass RC filter produces the following output signal:

$$
U(t)=\operatorname{Re}\left\{\frac{U_{0}}{R C} \int_{0}^{\infty} \exp \left[i \varphi\left(t-t^{\prime}\right)-t^{\prime} / R C\right] \mathrm{d} t^{\prime}\right\},
$$

which recalls the signals investigated in this paper [Eq. (18)].

Let us point out the possible applications of the studied effects. The pulses appear at the moments when the frequencies of the signal and reference beams coincide. This fact can be used in a Doppler scanning velocimeter estimating the unknown velocity from the time delay of the detected pulses. Moreover, the duration and shape of the pulses provide information about the sweep rate of the LFM signal, which can be caused by the uniformly accelerated motion of the object. This means that the velocity and acceleration can be measured simultaneously by detection of the only pulse, which is very actual in investigations of fast processes. In this work we used frequency shifts and sweep rates in ranges $|\Delta f|=0-10 \mathrm{MHz}$ and $|A|=0.0005-1000 \mathrm{MHz} / \mathrm{s}$. They would correspond to the following ranges of velocity and acceleration of the light reflecting object: $|V|=0-3 \mathrm{~m} / \mathrm{s}$ and $|a|=0.0003-600 \mathrm{~m} / \mathrm{s}^{2}$.

The devices realized on the considered effects will inherit the advantages of the devices using conventional non-steadystate photo-EMF and two-wave mixing effects. They include rather high sensitivity and adaptivity, i.e., the possibility of operation in the presence of a slow phase drift in the interferometric setup. The devices will also be able to detect light signals with complicated wave fronts, even with speckle patterns produced by reflection from the real diffusely scattering objects. However, these new methods will have some limitations typical for the standard techniques [33,34]: the light source should possess enough transverse and longitudinal coherence to form an interference pattern with maximum contrast; large spatial frequency $K$ may also be preferable, so that each speckle spot contains several interference fringes [35].

The developed techniques can also be used for characterization of widegap semiconductors. Instead of investigations of frequency transfer functions, we can measure the pulse response and estimate the space-charge formation time $\tau_{s c}$ and corresponding photoconductivity $\sigma_{0}$. Both the low and high sweep rates of the LFM signal can be used for estimation of $\tau_{s c}$ [see Eqs. (22) and (23)].

\section{CONCLUSION}

LFM of optical beams provides another way to excite timedependent photo-EMF and two-wave mixing signals. The effects reveal themselves as electrical and optical pulses arising in photoconductive and photorefractive media at the moments when the interference pattern slows down and stops. The duration, amplitude, and shape of the pulses are determined by the sweep rate of LFM and by the material parameters. This may allow us to develop new techniques of material characterization and detection of frequency modulated optical signals.

\section{ACKNOWLEDGMENTS}

M. B., V. K., and I. S. acknowledge the financial support from the Ministry of Knowledge Economy of Korea.

\section{REFERENCES}

1. P. Günter and J.-P. Huignard, Photorefractive Materials and their Applications II, Vol. 62 of Topics in Applied Physics (Springer, 1989).

2. M. P. Petrov, S. I. Stepanov, and A. V. Khomenko, Photorefractive Crystals in Coherent Optical Systems, Vol. 59 of Springer Series in Optical Sciences (Springer, 1991).

3. S. I. Stepanov, I. A. Sokolov, G. S. Trofimov, V. I. Vlad, D. Popa, and I. Apostol, "Measuring vibration amplitudes in the picometer range using moving light gratings in photoconductive GaAs:Cr," Opt. Lett. 15, 1239-1241 (1990).

4. R. K. Ing and J.-P. Monchalin, "Broadband optical detection of ultrasound by two-wave mixing in a photorefractive crystal," Appl. Phys. Lett. 59, 3233-3235 (1991).

5. C. C. Wang, F. Davidson, and S. Trivedi, "Simple laser velocimeter that uses photoconductive semiconductors to measure optical frequency differences," Appl. Opt. 34, 6496-6499 (1995).

6. C. C. Wang, R. A. Linke, D. D. Nolte, M. R. Melloch, and S. Trivedi, "Enhanced detection bandwidth for optical Doppler frequency measurements using moving space charge field effects in GaAs multiple quantum wells," Appl. Phys. Lett. 70, 2034-2036 (1997). 
7. D. M. Pepper, G. J. Dunning, D. D. Nolte, J. A. Coy, B. Pouet, G. D. Bacher, and M. B. Klein, "Improved responsivity of nonsteady-state photoinduced electromotive force sensors using asymmetric interdigitated contacts for laser-based ultrasound detection," Opt. Photon. News 10(12), 11-12 (1999).

8. P. Delaye, S. de Rossi, and G. Roosen, "Photorefractive vibrometer for the detection of high-amplitude vibrations on rough surfaces," J. Opt. A 2, 209-215 (2000).

9. A. A. Kamshilin, R. V. Romashko, and Y. N. Kulchin, "Adaptive interferometry with photorefractive crystals,” J. Appl. Phys. 105, 031101 (2009).

10. A. A. Kolegov, S. M. Shandarov, G. V. Simonova, L. A. Kabanova, N. I. Burimov, S. S. Shmakov, V. I. Bykov, and Y. F. Kargin, "Adaptive interferometry based on dynamic reflective holograms in cubic photorefractive crystals," Quantum Electron. 41, 847-852 (2011).

11. S. Mansurova, P. Moreno Zarate, P. Rodriguez, S. Stepanov, S. Köber, and K. Meerholz, "Non-steady-state photoelectromotive force effect under linear and periodical phase modulation: application to detection of Doppler frequency shift," Opt. Lett. 37, 383-385 (2012).

12. G. S. Trofimov and S. I. Stepanov, "Time-dependent holographic currents in photorefractive crystals," Sov. Phys. Solid State 28, 1559-1562 (1986).

13. M. P. Petrov, I. A. Sokolov, S. I. Stepanov, and G. S. Trofimov, "Non-steady-state photo-electro-motive force induced by dynamic gratings in partially compensated photoconductors," J. Appl. Phys. 68, 2216-2225 (1990).

14. I. A. Sokolov and S. I. Stepanov, "Non-steady-state photoelectromotive force in crystals with long photocarrier lifetimes," J. Opt. Soc. Am. B 10, 1483-1488 (1993).

15. N. V. Kukhtarev, T. Kukhtareva, S. F. Lyuksyutov, M. A. Reagan, P. P. Banerjee, and P. Buchhave, "Running gratings in photoconductive materials,” J. Opt. Soc. Am. B 22, 1917-1922 (2005).

16. U. Haken, M. Hundhausen, and L. Ley, "Analysis of the movingphotocarrier-grating technique for the determination of mobility and lifetime of photocarriers in semiconductors," Phys. Rev. B 51, 10579-10590 (1995)

17. J. P. Huignard and A. Marrakchi, "Coherent signal beam amplification in two-wave mixing experiments with photorefractive $\mathrm{Bi}_{12} \mathrm{SiO}_{20}$ crystals," Opt. Commun. 38, 249-254 (1981).

18. G. C. Valley, "Two-wave mixing with an applied field and a moving grating," J. Opt. Soc. Am. B 1, 868-873 (1984).

19. P. Refregier, L. Solymar, H. Rajbenbach, and J. P. Huignard "Two-beam coupling in photorefractive $\mathrm{Bi}_{12} \mathrm{SiO}_{20}$ crystals with moving grating: theory and experiments," J. Appl. Phys. 58, 45-57 (1985).

20. A. A. Kamshilin, J. Frejlich, and L. Cescato, "Photorefractive crystals for the stabilization of the holographic setup," Appl. Opt. 25, 2375-2381 (1986).
21. S. Bian and J. Frejlich, "Phase modulated two-wave mixing in crystals with long photocarrier lifetimes,” J. Mod. Opt. 43, 1185-1198 (1996).

22. H. Veenhuis, K. Buse, E. Krätzig, N. Korneev, and D. Mayorga, "Non-steady-state photoelectromotive force in reduced lithium niobate crystals,” J. Appl. Phys. 86, 2389-2392 (1999)

23. M. C. Gather, S. Mansurova, and K. Meerholz, "Determining the photoelectric parameters of an organic photoconductor by the photoelectromotive-force technique," Phys. Rev. B 75, 165203 (2007).

24. T. O. dos Santos, J. Frejlich, and K. Shcherbin, "Photo electromotive force in CdTe:Ge: manifestation of two photorefractive centers,” Appl. Phys. B 99, 701-707 (2010).

25. I. de Oliveira, A. A. Freschi, I. Fier, and J. Frejlich, "Stabilized photorefractive running holograms, with arbitrarily selected phase shift, for material characterization,” Opt. Mater. Express 2, 228-234 (2012).

26. G. S. Trofimov, S. I. Stepanov, M. P. Petrov, and M. V. Krasin'kova, "Time-varying photo-EMF associated with spatially nonuniform surface excitation of GaAs:Cr," Sov. Tech. Phys. Lett. 13, 108-109 (1987).

27. S. I. Stepanov and G. S. Trofimov, "Transient EMF in crystals having ambipolar photoconductivity," Sov. Phys. Solid State 31, 49-50 (1989).

28. N. A. Korneev and S. I. Stepanov, "Non-steady-state photoelectromotive force in semiconductor crystals with high light absorption,” J. Appl. Phys. 74, 2736-2741 (1993).

29. N. Korneev, S. Mansurova, and S. Stepanov, "Nonstationary current in bipolar photoconductor with slow photoconductivity relaxation," J. Appl. Phys. 78, 2925-2931 (1995).

30. G. Pauliat and G. Roosen, "Theoretical and experimental study of diffraction in optically active and linearly birefringent sillenite crystals," Ferroelectrics 75, 281-294 (1987).

31. A. M. Plesovskikh, S. M. Shandarov, A. G. Mart'yanov, A. E Mandel, N. I. Burimov, E. A. Shaganova, Y. F. Kargin, V. V. Volkov, and A. V. Egorysheva, "Vector two-wavelength interaction on reflection holographic gratings in cubic gyrotropic photorefractive crystals," Quantum Electron. 35, 163-168 (2005).

32. R. V. Romashko, S. Di Girolamo, Y. N. Kulchin, and A. A. Kamshilin, "Photorefractive vectorial wave mixing in different geometries," J. Opt. Soc. Am. B 27, 311-317 (2010).

33. R. J. Collier, C. B. Burckhardt, and L. H. Lin, Optical Holography (Academic, 1971).

34. R. Jones and C. Wykes, Holographic and Speckle Interferometry (Cambridge University, 1989).

35. I. A. Sokolov, P. Hess, M. A. Bryushinin, V. V. Kulikov, S. H. Khan, and K. T. V. Grattan, Interferometry in Speckle Light: Theory and Applications, P. Jacquot and J.-M. Fournier, eds. (Springer, 2000), pp. 187-194. 